\title{
Stationary Flow of Blood in a Rigid Vessel in the Presence of an External Magnetic Field: Considerations about the Forces and Wall Shear Stresses
}

\author{
Agnès Drochon', Vincent Robin², Odette Fokapu1, Dima Abi-Abdallah Rodriguez ${ }^{3}$ \\ ${ }^{1}$ UMR CNRS 7338, Université de Technologie de Compiègne, Galileo Galilei Sorbonne Universités, Compiègne, \\ France \\ ${ }^{2}$ LMAC, Université de Technologie de Compiègne, Galileo Galilei Sorbonne Universités, Compiègne, France \\ ${ }^{3}$ IR4M, Université Paris Sud-Paris, Université Paris-Saclay, Orsay, France \\ Email: agnes.drochon@utc.fr
}

Received 27 November 2015; accepted 2 February 2016; published 5 February 2016

Copyright (C) 2016 by authors and Scientific Research Publishing Inc.

This work is licensed under the Creative Commons Attribution International License (CC BY). http://creativecommons.org/licenses/by/4.0/

(c) (i) Open Access

\section{Abstract}

The magnetohydrodynamics laws govern the motion of a conducting fluid, such as blood, in an externally applied static magnetic field $B_{0}$. When an artery is exposed to a magnetic field, the blood charged particles are deviated by the Lorentz force thus inducing electrical currents and voltages along the vessel walls and in the neighboring tissues. Such a situation may occur in several biomedical applications: magnetic resonance imaging (MRI), magnetic drug transport and targeting, tissue engineering... In this paper, we consider the steady unidirectional blood flow in a straight circular rigid vessel with non-conducting walls, in the presence of an exterior static magnetic field. The exact solution of Gold (1962) (with the induced fields not neglected) is revisited. It is shown that the integration over a cross section of the vessel of the longitudinal projection of the Lorentz force is zero, and that this result is related to the existence of current return paths, whose contributions compensate each other over the section. It is also demonstrated that the classical definition of the shear stresses cannot apply in this situation of magnetohydrodynamic flow, because, due to the existence of the Lorentz force, the axisymmetry is broken.

\section{Keywords}

Magnetohydrodynamic Flow of Blood, Wall Shear Stresses, Magnetic Field in Biomedical Applications 


\section{Introduction}

The magnetohydrodynamics laws govern the motion of a conducting fluid, such as blood, in an externally applied static magnetic field $B_{0}$. When an artery is exposed to a magnetic field, the blood charged particles are deviated by the Lorentz force thus inducing electrical currents and voltages along the vessel walls and in the neighboring tissues. Such a situation may occur in several biomedical applications: magnetic resonance imaging (MRI) [1]-[5], magnetic drug transport and targeting [6]-[9], tissue engineering [10]-[13]...

An optimal modelisation of the magnetohydrodynamic flow of blood should include the pulsatility of flow, the deformability and conductivity of the vessel wall, together with the induced electrostatic and electromagnetic fields. This leads to a complex mathematical problem and analytical solutions may be found only under restrictive hypotheses.

The analysis presented in this paper is based on the exact solution given by Gold [14] for the stationary blood flow in a rigid vessel with insulating wall in the presence of an external static magnetic field. This analysis completes a previous one [15]. Since the magnetic field breaks the flow axisymmetry, it is interesting to study in details the velocities, pressure, forces, induced fields and currents in a vessel cross section. This is the aim of this paper. Such integrations over the cross-section of the vessel would also be necessary in order to explore the feasibility of deriving 1-D equations [16] for this type of flow.

\section{Position of the Problem}

As explained by Gold [14] and by Abi-Abdallah et al. [15], the Navier-Stokes equations including the Lorentz force $\mathbf{j} \wedge \mathbf{B}$ (1), coupled with the induction Equation (2) govern the flow of a conducting, incompressible, Newtonian fluid in an externally applied static magnetic field $B_{0}$.

$$
\begin{gathered}
\rho\left(\frac{\partial \overrightarrow{\mathbf{u}}}{\partial t}+(\overrightarrow{\mathbf{u}} \cdot \vec{\nabla}) \overrightarrow{\mathbf{u}}\right)=-\operatorname{gradP}+\eta \Delta \overrightarrow{\mathbf{u}}+\frac{1}{\mu}(\operatorname{curl} \overrightarrow{\mathbf{B}}) \wedge \overrightarrow{\mathbf{B}} \\
\frac{\partial \overrightarrow{\mathbf{B}}}{\partial t}=\operatorname{curl}(\overrightarrow{\mathbf{u}} \wedge \overrightarrow{\mathbf{B}})+\frac{1}{\sigma \mu} \Delta \overrightarrow{\mathbf{B}}
\end{gathered}
$$

where $\mathbf{u}$ and $P$ are the fluid velocity and pressure, $t$ is the time, $\mathbf{j}=(\operatorname{curl} \mathbf{B}) / \mu$ is the electric current density; $\mu$ is the magnetic permeability; $\rho, \eta$ and $\sigma$ are the fluid density, viscosity and conductivity. The magnetization force that could be induced in the blood by magnetic field gradients is neglected in this study since $B_{0}$ is uniform and the induced magnetic field $B_{I}(r, \theta)$ is very small [17].

Using the identity:

$$
(\operatorname{curl} \overrightarrow{\mathbf{B}}) \wedge \overrightarrow{\mathbf{B}}=-\frac{1}{2} \operatorname{grad}\left(\|\overrightarrow{\mathbf{B}}\|^{2}\right)+(\overrightarrow{\mathbf{B}} \cdot \vec{\nabla}) \overrightarrow{\mathbf{B}}
$$

it is possible to write (1) in the form:

$$
\rho\left(\frac{\partial \overrightarrow{\mathbf{u}}}{\partial t}+(\overrightarrow{\mathbf{u}} \cdot \vec{\nabla}) \overrightarrow{\mathbf{u}}\right)=-\operatorname{grad}\left[P+\frac{1}{2 \mu}\|\overrightarrow{\mathbf{B}}\|^{2}\right]+\eta \Delta \overrightarrow{\mathbf{u}}+\frac{1}{\mu}(\overrightarrow{\mathbf{B}} \cdot \vec{\nabla}) \overrightarrow{\mathbf{B}}
$$

thus introducing the magnetic pressure $\frac{1}{2 \mu}\|\overrightarrow{\mathbf{B}}\|^{2}$.

\section{The Case of a Unidirectional Steady Blood Flow in a Rigid Circular Vessel with Insulating Walls}

Gold [14] considered the case of a unidirectional steady blood flow in a rigid circular vessel with insulating walls (Figure 1, from Abi-Abdallah et al. [15]). The velocity and magnetic field are defined in the cylindrical frame $\left(\mathbf{e}_{r}, \mathbf{e}_{\theta}, \mathbf{e}_{z}\right)$ as:

$$
\overrightarrow{\mathbf{u}} \mid \begin{gathered}
0 \\
0 \\
u_{z}(r, \theta)
\end{gathered} \quad \text { and } \overrightarrow{\mathbf{B}} \mid \begin{gathered}
B_{0} \cos \theta \\
-B_{0} \sin \theta \\
B_{I}(r, \theta)
\end{gathered}
$$




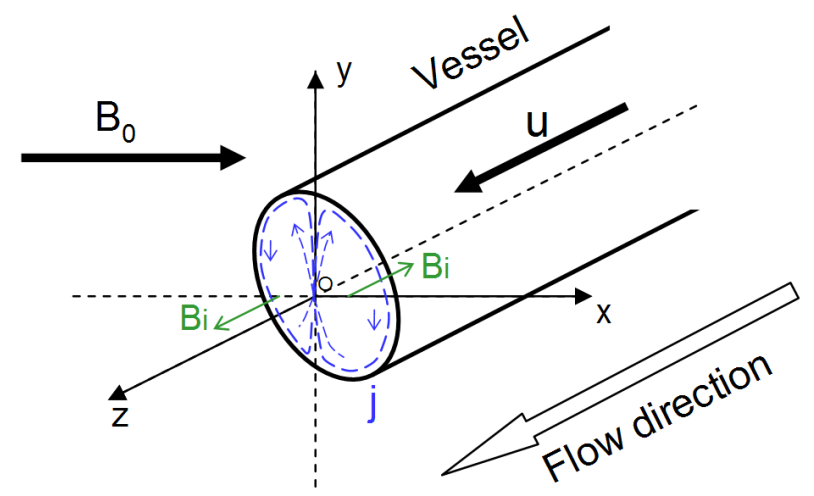

Figure 1. Inductions in the vessel. The induced currents circulate in a closed loop in the transverse plane. The induced field is parallel to the $\mathrm{Oz}$ axis with opposite directions on each side of $\mathrm{Oy}$.

The induced magnetic field is parallel to the flow and guarantees $\operatorname{div} \mathbf{B}=0$. The continuity equation $\operatorname{div} \mathbf{u}=0$ is also satisfied. The charge separation is supposed to arise in a plane which is perpendicular to the velocity, thus inducing an electric field that is located in that same plane. The induced current in the tube center is oriented upward as shown, and the return currents adjacent to the walls are oriented downward. In the tube center, $\mathbf{j}$ and $\mathbf{B}$ are directed so that the ponderomotive force is opposed to the fluid flow. Near the walls, however, the return currents are in the opposite direction, thus the fluid flow is enhanced by the ponderomotive force that is generated by the return currents. The currents cannot escape the vessel because the wall is insulating. The induced magnetic field results from these induced currents flowing in closed loops (Biot and Savart law).

The projection of (1) in the cylindrical frame is thus:

$$
\begin{aligned}
& -\frac{\partial P}{\partial r}-\frac{1}{\mu} B_{I} \frac{\partial B_{I}}{\partial r}=0 \\
& -\frac{1}{r} \frac{\partial P}{\partial \theta}-\frac{1}{\mu} \frac{B_{I}}{r} \frac{\partial B_{I}}{\partial \theta}=0 \\
& -\frac{\partial P}{\partial z}+\eta\left(\frac{1}{r} \frac{\partial u_{z}}{\partial r}+\frac{\partial^{2} u_{z}}{\partial r^{2}}+\frac{1}{r^{2}} \frac{\partial^{2} u_{z}}{\partial \theta^{2}}\right)+\frac{1}{\mu}\left(B_{0} \cos \theta \frac{\partial B_{I}}{\partial r}-\frac{B_{0} \sin \theta}{r} \frac{\partial B_{I}}{\partial \theta}\right)=0
\end{aligned}
$$

The projections on $\mathbf{e}_{r}$ and $\mathbf{e}_{\theta}$ (6a) and (6b) may also be written:

$$
\mid \begin{aligned}
& \frac{\partial P_{\text {total }}}{\partial r}=0 \\
& \frac{\partial P_{\text {total }}}{\partial \theta}=0
\end{aligned} \quad \text { with } P_{\text {total }}=P+\frac{1}{2 \mu}\|\overrightarrow{\mathbf{B}}\|^{2} \text { and }\|\overrightarrow{\mathbf{B}}\|^{2}=B_{0}^{2}+B_{I}^{2}
$$

It appears from (7) that $P_{\text {total }}$ is uniform in a transverse section of the vessel (whereas $P$ is not) and that

$$
\frac{\partial P_{\text {total }}}{\partial z}=\frac{\partial P}{\partial z}
$$

Of course, the projection of (4) also leads to (6)-(8).

The induction Equation (2) has only one interesting projection: the longitudinal one (along $\mathbf{e}_{2}$ ):

$$
0=B_{0}\left(\cos \theta \frac{\partial u_{z}}{\partial r}-\frac{\sin \theta}{r} \frac{\partial u_{z}}{\partial \theta}\right)+\frac{1}{\sigma \mu}\left(\frac{1}{r} \frac{\partial B_{I}}{\partial r}+\frac{\partial^{2} B_{I}}{\partial r^{2}}+\frac{1}{r^{2}} \frac{\partial^{2} B_{I}}{\partial \theta^{2}}\right)
$$

\section{Integration of the Equations over a Transverse Section of the Vessel}

We are now interested in integrating the longitudinal projection in (6) and (9) over a transverse section of the vessel. 
Using the identity (A1) established in Appendix A, it is possible to show that:

$$
\int_{0}^{R} \int_{0}^{2 \pi}\left(\cos \theta \frac{\partial B_{I}}{\partial r}-\frac{\sin \theta}{r} \frac{\partial B_{I}}{\partial \theta}\right) \mathrm{d} \theta r \mathrm{~d} r=0
$$

because,

$$
\int_{0}^{R} \int_{0}^{2 \pi}\left(\cos \theta \frac{\partial B_{I}}{\partial r}-\frac{\sin \theta}{r} \frac{\partial B_{I}}{\partial \theta}\right) \mathrm{d} \theta r \mathrm{~d} r=\int_{0}^{2 \pi} R B_{I}(R, \theta) \cos \theta \mathrm{d} \theta
$$

and $B_{I}(R, \theta)=0$ for a non-conducting wall.

Equation (10) means that the integration over a cross section of the vessel of the longitudinal projection of the Lorentz force is zero:

$$
\int_{0}^{R} \int_{0}^{2 \pi}\left[(\vec{j} \wedge \vec{B}) \cdot \vec{e}_{z}\right] \mathrm{d} \theta r \mathrm{~d} r=0
$$

This seems related to the existence of current return paths (Figure 1). The contributions of these currents compensate each other over the section, and the total contribution is null. In the case of neglected induced fields or electrically conducting channels, the recirculating loops do not exist and this result is no longer valid.

Using the same identity (A1; Appendix A), it is also possible to show that:

$$
\int_{0}^{R} \int_{0}^{2 \pi}\left(\cos \theta \frac{\partial u_{z}}{\partial r}-\frac{\sin \theta}{r} \frac{\partial u_{z}}{\partial \theta}\right) \mathrm{d} \theta r \mathrm{~d} r=0
$$

because,

$$
\int_{0}^{R} \int_{0}^{2 \pi}\left(\cos \theta \frac{\partial u_{z}}{\partial r}-\frac{\sin \theta}{r} \frac{\partial u_{z}}{\partial \theta}\right) \mathrm{d} \theta r \mathrm{~d} r=\int_{0}^{2 \pi} R u_{z}(R, \theta) \cos \theta \mathrm{d} \theta
$$

and $u_{z}(R, \theta)=0$ (no-slip condition at the rigid wall).

Integration of (6c) and (9) over a cross section of the vessel thus yields:

$$
\left\{\begin{array}{l}
\left(-\frac{\partial P}{\partial z}\right) \pi R^{2}+\eta \iint_{A}\left(\Delta u_{z}\right) \mathrm{d} A=0 \\
\iint_{A}\left(\Delta B_{I}\right) \mathrm{d} A=0
\end{array}\right.
$$

This shows that the integral of $\Delta u_{z}$ over the section is independent of $B_{0}$, whereas the mean velocity (integral of $u_{z}$ over the section) depends on $B_{0}$ (see Graphs no. 5 and no. 18 in Abi-Abdallah et al. [15]).

Using the identity (A2) of the Appendix A, (15) become:

$$
\begin{aligned}
& \eta R \int_{0}^{2 \pi} \frac{\partial u_{z}}{\partial r}(R, \theta) \mathrm{d} \theta=\pi R^{2}\left(\frac{\partial P}{\partial z}\right) \\
& R \int_{0}^{2 \pi} \frac{\partial B_{I}}{\partial r}(R, \theta) \mathrm{d} \theta=0
\end{aligned}
$$

Considering that: $\overrightarrow{\mathbf{j}}=\frac{1}{\mu} \operatorname{curl} \overrightarrow{\mathbf{B}}$, we have:

$$
\frac{\partial B_{I}}{\partial r}=-\mu j_{\theta}
$$

and (17) means that:

$$
-R \mu \int_{0}^{2 \pi} j_{\theta}(R, \theta) \mathrm{d} \theta=0
$$

This result can be checked in the Graphs no. 10 of Abi-Abdallah et al. [15] and is coherent with the current compensation that is illustrated in Figure 1 of this paper.

We can also notice that, in a hypothetic case where the gradient of velocity at the wall would not depend on $\theta$, 
(16) would reduce to a classical balance of forces on a small volume element of unit length:

$$
2 \pi R \eta \frac{\partial u_{z}}{\partial r}(R)=\pi R^{2}\left(\frac{\partial P}{\partial z}\right)
$$

Equation (16) thus demonstrates that the statements of the viscous stresses have to be reconsidered in the case of MHD flow, because the velocity $u_{z}$ depends on $\mathrm{r}$ and on $\theta$. This can be understood looking at the Graphs no. 3 in Abi-Abdallah et al. [15]. This Figure presents the iso-velocity lines in a transversal plane of the vessel. One can observe that the presence of a static magnetic field reduces the blood flow and flattens the velocity profile. This appears more clearly for $B_{0}>20 \mathrm{~T}$. The profile is stretched parallel to the direction of $B_{0}(\theta=0)$. The iso-velocity lines are more tightened in the position $\theta=0$ than in the position $\theta=\pi / 2$, indicating that the velocity gradient $\partial u_{z} / \partial r$ is higher for $\theta=0$ than for $\theta=\pi / 2$. This gradient is also higher when $B_{0}$ increases (in the position $\theta=0$, the lines are more and more tightened for higher values of $B_{0}$ ). The same conclusions can be drawn when looking at the Graphs no. 4 in Abi-Abdallah et al. [15]. In this figure, it appears more clearly that the velocity profile becomes more and more flat when $B_{0}$ increases, with thin boundary layers (called "Hartmann boundary layers") at the walls, where viscous drag drives the flow to zero. It is in the Hartmann layers that the electric currents, induced at the core flow in the y-direction, return and close the current loop. In an electrically insulated channel, the modification of the parabolic laminar velocity profile increases shear friction at the walls, which in turn increases $(\partial P / \partial z)$. This is what (16) shows.

It could be possible to confirm these qualitative observations by calculating $\partial u_{z} / \partial r$ and $\partial u_{z} / \partial \theta$ from Equation (14) of Abi-Abdallah et al. [15]. Evaluating these quantities at the wall should allow to see whether the stresses reach some levels that could be at risk: 1) for the vessel wall (plaque rupture in case of atherosclerotic lesion [18], severity of some aneurysms [19], ...), 2) for the mechanotransduction in the endothelial cells, 3) for other cells attachment and/or transmigration (white blood cells, tumor cells, cells seeded in vascular substitutes [20], ...). Estimation of shear stresses at the wall may also have important implications in magnetic nanoparticle delivery and drug targeting [6] [21].

\section{Conclusion}

In this paper, we have considered the steady unidirectional blood flow in a circular rigid vessel with insulating walls, in the presence of an exterior magnetic field. The exact solution of Gold [14] (with the induced fields not neglected) has been explored. It has been shown that the integration over a cross section of the vessel of the longitudinal projection of the Lorentz force is zero, and that this result is related to the existence of current return paths, whose contributions compensate each other over the section. It has been also demonstrated that the classical definition of the shear stresses cannot apply in this situation of magnetohydrodynamic flow, because, due to the existence of the Lorentz force, the axisymmetry is broken.

\section{Acknowledgements}

The authors would like to thank Professor Yuri Molodtsof, from University of Technology of Compiègne (UTC), for his helpful comments on this work.

\section{Competing Interests}

None.

\section{Ethical Approval}

Not required.

\section{References}

[1] Laffon, E., Marthan, R., Montaudon, M., Latrabe, V., Laurent, F. and Ducassou, D. (2005) Feasibility of Aortic Pulse Pressure and Pressure Wave Velocity MRI Measurement in Young Adults. Journal of Magnetic Resonance Imaging, 21, 53-58. http://dx.doi.org/10.1002/jmri.20227

[2] Nijm, G., Swiryn, S., Larson, A. and Sahakian, A. (2008) Blood Flow Potential from the Surface Electrocardiogram in Magnetic Resonance Imaging. Medical \& Biological Engineering \& Computing, 46, 729-733. 
http://dx.doi.org/10.1007/s11517-008-0307-1

[3] Ibrahim, E.S., Johnson, K., Miller, A., Shaffer, J. and White, R. (2010) Measuring Aortic Pulse Wave Velocity using High-Field Cardiovascular Magnetic Resonance: Comparison of Techniques. Journal of Cardiovascular Magnetic Resonance, 12, 26-39. http://dx.doi.org/10.1186/1532-429X-12-26

[4] Kyriakou, A., Neufeld, E., Szczerba, D., Kainz, W., Luechinger, R., Kozerke, S., Mc Gregor, R. and Kuster, N. (2012) Patient-Specific Simulations and Measurements of the Magneto-Hemodynamic Effect in Human Primary Vessels. Physiological Measurements, 33, 117-130. http://dx.doi.org/10.1088/0967-3334/33/2/117

[5] Krug, K., Rose, G., Clifford, G. and Oster, J. (2013) ECG-Based Gating in Ultra High Field Cardiovascular Magnetic Resonance Using an Independent Component Analysis Approach. Journal of Cardiovascular Magnetic Resonance, 15, 104-116. http://dx.doi.org/10.1186/1532-429X-15-104

[6] Kenjeres, S. (2008) Numerical Analysis of Blood Flow in Realistic Arteries Subjected to Strong Non-Uniform Magnetic Fields. International Journal of Heat and Fluid Flow, 29, 752-764. http://dx.doi.org/10.1016/j.ijheatfluidflow.2008.02.014

[7] Ciofani, G., Riggio, C., Raffa, V., Menciassi, A. and Cuschieri, A. (2009) A Bi-Modal Approach Against Cancer: Magnetic Alginate Nanoparticles for Combined Chemotherapy and Hyperthermia. Medical Hypotheses, 73, 80-82. http://dx.doi.org/10.1016/j.mehy.2009.01.031

[8] Morega, A., Dobre, A. and Morega, M. (2011) Magnetic Field-Flow Interactions in Drug Delivery through an Arterial System. Revue Roumaine des Sciences Techiques_Electrotechnique et Energetique, 56, 199 -208.

[9] Riegler, J., Lau, K., Garcia-Prieto, A., Price, A., Richards, T., Pankhurst, Q. and Lythgoe, M. (2011) Magnetic Cell Delivery for Peripheral Arterial Disease: A Theoretical Framework. Medical Physics, 38, 3932-3943. http://dx.doi.org/10.1118/1.3593363

[10] Perea, H., Aigner, J., Hopfner, U. and Wintermantel, E. (2006) Direct Magnetic Tubular Cell Seeding: A Novel Approach for Vascular Tissue Engineering. Cells Tissues Organs, 183, 156 -165. http://dx.doi.org/10.1159/000095989

[11] Perea, H., Aigner, J., Heverhagen, J.T., Hopfner, U. and Wintermantel, E. (2007) Vascular Tissue Engineering with Magnetic Nanoparticles: Seeing Deeper. Journal of Tissue Engineering and Regenerative Medicine, 1, 318-321. http://dx.doi.org/10.1002/term.32

[12] Xu, H., Othman, S. and Magin, R. (2008) Monitoring Tissue Engineering Using Magnetic Resonance Imaging. Journal of Biosciences and Bioengineering, 106, 515-527. http://dx.doi.org/10.1263/jbb.106.515

[13] Poirier-Quinot, M., Frasca, G., Wilhelm, C., Luciani, N., Ginefri, J.C., Darrasse, L., Letourneur, D., Le Visage, C. and Gazeau, F. (2010) High Resolution 1.5 Tesla Magnetic Resonance Imaging for Tissue-Engineered Constructs: A Noninvasive Tool to Assess Three-Dimensional Scaffold Architecture and Cell Seeding. Tissue Engineering Part C Methods, 16, 185-200. http://dx.doi.org/10.1089/ten.tec.2009.0015

[14] Gold, R. (1962) Magnetohydrodynamic Pipe Flow. Journal of Fluid Mechanics, 13, 505-512. http://dx.doi.org/10.1017/S0022112062000889

[15] Abi-Abdallah, D., Drochon, A., Robin, V. and Fokapu, O. (2009) Effects of Static Magnetic Field Exposure on Blood Flow. The European Physical Journal Applied Physics, 45, 11301-11317. http://dx.doi.org/10.1051/epjap:2008193

[16] Olufsen, M. (2000) Numerical Simulation and Experimental Validation of Blood Flow in Arteries with Structured Tree Outflow Conditions. Annals of Biomedical. Engineering, 28, 1281-1299. http://dx.doi.org/10.1114/1.1326031

[17] Tzirtzilakis, E. (2005) A Mathematical Model for Blood Flow in Magnetic Field. Physics of Fluids, 17, Article ID: 077103. http://dx.doi.org/10.1063/1.1978807

[18] Weddell, J., Kwack, J., Imoukhuede, P. and Masud, A. (2015) Hemodynamic Analysis in an Idealized Artery Tree: Differences in Wall Shear Stress between Newtonian and Non-Newtonian Blood Models. PLoS ONE, 10, e0124575. http://dx.doi.org/10.1371/journal.pone.0124575

[19] Boussel, L., Rayz, V., Martin, A., Acevedo-Bolton, G., Lawton, M., Higashida, R., Smith, W., Young, W. and Saloner, D. (2009) Phase-Contrast MRI Measurements in Intra-Cranial Aneurysms In-Vivo of Flow Patterns, Velocity Fields and Wall Shear-Stress: A Comparison with CFD. Magnetic Resonance in Medicine, 61, 409-417. http://dx.doi.org/10.1002/mrm.21861

[20] Castro, E. and Mano, J. (2013) Magnetic Force-Based Tissue Engineering and Regenerative Medicine. Journal of Biomedical Nanotechnology, 9, 1129-1136. http://dx.doi.org/10.1166/jbn.2013.1635

[21] Sensenig, R., Sapir, Y., MacDonald, C., Cohen, S. and Polyak, B. (2012) Magnetic Nanoparticle-Based Approaches to Locally Target Therapy and Enhance Tissue Regeneration in Vivo. Nanomedecine, 7, 1425-1442. http://dx.doi.org/10.2217/nnm.12.109 


\section{Appendix A}

For every smooth function $\mathrm{f}(\mathrm{r}, \theta)$, it is possible to establish the following two identities: Identity (A1): $\int_{0}^{R} \int_{0}^{2 \pi}\left(\cos \theta \frac{\partial f}{\partial r}-\frac{\sin \theta}{r} \frac{\partial f}{\partial \theta}\right) \mathrm{d} \theta r \mathrm{~d} r=\int_{0}^{2 \pi} R f(R, \theta) \cos \theta \mathrm{d} \theta$

Proof:

$$
\begin{aligned}
& \int_{0}^{R} \int_{0}^{2 \pi}\left(\cos \theta \frac{\partial f}{\partial r}-\frac{\sin \theta}{r} \frac{\partial f}{\partial \theta}\right) \mathrm{d} \theta r \mathrm{~d} r \\
& =\int_{0}^{2 \pi} \cos \theta\left(\int_{0}^{R} r \frac{\partial f}{\partial r} \mathrm{~d} r\right) \mathrm{d} \theta+\int_{0}^{R}\left(\int_{0}^{2 \pi}(-\sin \theta) \frac{\partial f}{\partial \theta} \mathrm{d} \theta\right) \mathrm{d} r \\
& =\int_{0}^{2 \pi} \cos \theta\left(\int_{0}^{R} r \frac{\partial f}{\partial r} \mathrm{~d} r\right) \mathrm{d} \theta+\int_{0}^{R}\left(\int_{0}^{2 \pi} \cos \theta f \mathrm{~d} \theta\right) \mathrm{d} r \\
& =\int_{0}^{2 \pi} \cos \theta\left(\int_{0}^{R}\left(r \frac{\partial f}{\partial r}+f\right) \mathrm{d} r\right) \mathrm{d} \theta \\
& =\int_{0}^{2 \pi} \cos \theta\left(\int_{0}^{R} \frac{\partial}{\partial r}(r f) \mathrm{d} r\right) \mathrm{d} \theta \\
& =\int_{0}^{2 \pi} \cos \theta R f(R, \theta) \mathrm{d} \theta
\end{aligned}
$$

Identity (A2): $\int_{0}^{2 \pi} \int_{0}^{R} \Delta f(r, \theta) \mathrm{d} r r \mathrm{~d} \theta=R \int_{0}^{2 \pi} \frac{\partial f}{\partial r}(R, \theta) \mathrm{d} \theta$

Proof:

$$
\begin{aligned}
& \int_{0}^{2 \pi} \int_{0}^{R} \Delta f(r, \theta) \mathrm{d} r r \mathrm{~d} \theta=\int_{0}^{2 \pi} \int_{0}^{R}\left(\frac{1}{r} \frac{\partial f}{\partial r}+\frac{\partial^{2} f}{\partial r^{2}}+\frac{1}{r^{2}} \frac{\partial^{2} f}{\partial \theta^{2}}\right) r \mathrm{~d} r \mathrm{~d} \theta \\
& =\int_{0}^{2 \pi}\left(\int_{0}^{R} \frac{\partial f}{\partial r} \mathrm{~d} r\right) \mathrm{d} \theta+\int_{0}^{2 \pi}\left(\int_{0}^{R} \frac{\partial^{2} f}{\partial r^{2}} r \mathrm{~d} r\right) \mathrm{d} \theta+\int_{0}^{R} \frac{1}{r}\left(\int_{0}^{2 \pi} \frac{\partial^{2} f}{\partial \theta^{2}} \mathrm{~d} \theta\right) \mathrm{d} r \\
& =\int_{0}^{2 \pi}\left(\int_{0}^{R} \frac{\partial}{\partial r}\left(r \frac{\partial f}{\partial r}\right) \mathrm{d} r\right) \mathrm{d} \theta+\int_{0}^{R} \frac{1}{r}\left(\int_{0}^{2 \pi} \frac{\partial^{2} f}{\partial \theta^{2}} \mathrm{~d} \theta\right) \mathrm{d} r \\
& =\int_{0}^{2 \pi} R \frac{\partial f}{\partial r}(R, \theta) \mathrm{d} \theta
\end{aligned}
$$

